Letras, Lima 48 (84-85): 41-48, 1976.

\title{
Intervención en los procesos y modificación del futuro
}

\author{
ARSENIO GUZMAN JORQUERA
}

Entre las nociones que intuitivamente se tiene sobre el tiempo (1), figura como una de las más destacadas la de que el pasado $\mathrm{y}$ el futuro pueden ser suficientemente distinguidos entre sí apelando a la convicción de que se da ante nosotros el pasado como algo cerrado y concluido, en el sentido de ser cada uno de sus eventos definitivamente inmodificable, mientras que el futuro se nos aparece como una infinidad abierta de posibilidades, de manera que éste, a diferencia del pasado, no es algo concluido ni mucho menos cerrado; cel futuro, se piensa, puede ser modificado. Es claro que lo centrgl gira qquí en torno a la concepción de un futuro modificable en contraste con un pasado inmodificable, no siendo, por tanto, la idea de lo "concluido" y las connotaciones afectivas que ella tenga verdaderamente significativas respecto del punto central que constituye el núcleo de la mentada diferencia entre pasado y futuro.

Es sobradamente conocido el hecho de que se piensa en que es posible modificar el futuro mediante intervenciones en los procesos, mientras que, de otro lado, se afirma que ninguna intervención podría ser capaz de modificar el pasado, de modo que los su. cesos pasados, como tales, resultan ser inalterables.

* Desarrollo de un fragmento de la "Introducción" de un libro en elaboración (aún sin título), sobre el tiempo y la historia. El ensayo que aquí se publica se presentó como ponencia a la Sociedad Peruana de Filosofía. Fue expuesto y discutido a lo largo de tres sesiones (julio-agosto de 1976).

(1) Puede encontrarse una exposición detallada de ésta y de otras nociones en Hans Reichenbach, El sentido del tiempo, México, Universidad Nacional Autónoma de México, 1959, p. 37 ss. 
La idea de que el pasado es inmodificable es indudablemente correcta. Adviértase que al usar aquí el término "pasado", lo hacemos para referirnos a los hechos ya ocurridos (conocidos o no), no a lo que en diversos informes puede ser dicho sobre tales hechos. Es obvio que, si de informes se trata, nada se opone a que éstos puedan ser cambiados; pero tal no es el caso tratándose de los hechos mismos; en estos términos, no es inapropiado admitir que el pasado no puede ser modificado. Esto no constituye, por tanto, al menos desde nuestro punto de vista actual, un problema que requiera un especial esclarecimiento.

La suposición de que el futuro es modificable, por otra parte, entraña particulares dificultades, las que en nuestra opinión merecen un tratamiento más cuidadoso, por cuanto lo aquí implicado, vale decir, la intervención en los procesos, no es algo suficientemente claro, ni mucho menos simple, por lo menos no tan simple que pueda ser admitido sin un previo análisis crítico.

En lo que sigue nos ocuparemos del problema planteado por la suposición de que mediante intervenciones en los procesos es posible modificar el futuro; trataremos de establecer en qué medida ( $\mathrm{si}$ lo es en alguna) puede ser adecuado suponer que es dable intervenir en los procesos, para determinar, sobre esa base, cuanto hay de correcto en la idea de que el futuro puede ser cambiado. Por lo demás, los análísis se limitarán al ámbito del mundo físico.

Si tomamos la secuencia $A-B-C-D$ para representar un proceso, al que denominaremos proceso $1, y$ la secuencia $A-B-C X-E$ para representar otro proceso, al que denominaremos proceso 2 y admitimos que tales secuencias, en su condición de representantes de procesos, representan igualmente secuencias temporales, podemos considerar que en ambos casos vamos de lo anterior a lo posterior, $y$ en tal sentido, en $1 \mathrm{D}$ resulta ser futuro respecto de $C$, de $B, 0$ de $A, y$ en $2 E$ es futuro respecto de $C X$, de $B$, o de $A$ (2). En los términos propuestos, 1 representa un proceso cualquiera sobre el que no se ha realizado ninguna intervención; su secuencia, por tanto, comienza en $A$ y concluye en $D$ (comienza y concluye relativamente hablando, los procesos en realidad no son tan independientes ni tan lineales; sin embargo, para los esclarecimientos que nos proponemos hacer tal simplificación no es en absoluto problemática, lo que diremos de los procesos simplificados en los mentados esquemas vale para cualquier proceso real, sea cual fuere el grado de su complejidad). El proceso representado en 2 puede ser considerado como un ejem-

(2) $\mathrm{Si}$ interpretamos estas secuencias como cadenas causales, cada evento será considerado como causa de los que le siguen. En este caso, Io que se considera intervención puede ser entendido como un fac-
tor causal más. 
plo que muestra el caso de una intervención en l, siendo, en este sentido, el evento representado por $\mathrm{X}$ el factor que constituye la intervención; como consecuencia de la intervención la secuencia concluye en $\mathrm{E}$, pese a haber comenzado en $\mathrm{A}$ y haber continuado en B. Es fácil pasar a pensar aquí cuál hubiera sido el evento final de la secuencia que comenzaba en $A$ si no se hubiera producido la intervención $\mathrm{X}$ : si no se hubiera producido la intervención $\mathrm{X}$, hubiera ocurrido $\mathrm{D}$. Estamos aquí construyendo un condicional contrafáctico ( 3 ), y si quisiéramos probar este peculiar condicional podría parecernos suficiente realizar el proceso en diversas condiciones (4). Se supondría, en el caso de los esquemas que estamos utilizando, que bastaría, para probar el contrafáctico en cuestión, dejar la secuencia iniciada en A marchar hasta su conclusión en $D$, pensando que se llega a $D$ precisamente por no haber intervenido $\mathrm{X}$.

Puesto que en la prueba mostrada se estaría presuponiendo que el pasado permanece inalterable, pudiera parecer que si se piensa en términos de "tiempo positivo" y "tiempo negativo" la situación sería más compleja, por cuanto en un "tiempo negativo" sería futuro lo primeramente llamado pasado (en el "tiempo positivo") y viceversa, con lo que vendría a resultar posible modificar el pasado (5), siendo, por lo demás, inmodificable lo considerado futuro en el "tiempo positivo". Es claro que esto sería algo más que una mera fantasía si fuera el caso de darse realmente un "tiemnegativo". Ahora bien, consideramos inconveniente e infundado hablar sobre lo real en términos de "tiempo negativo". Procederemos a examinar con más detenimiento este punto.

Si dos procesos dados muestran en sus eventos las secuencias $A-B-C$ y $C-B$ - $\mathrm{A}$ A p nolnecesdriamentestienen que ser éstas entendidas como representantes de dos direcciones temporales, una "negativa" y la otra "positiva", pues si quisiéramos pensar que $A-B-C$ marchara "hacia adelante" (positivamente) de $A$ hacia $C$, y $C-B-A$ marchara "hacia atrás", de $C$ hacia $A$, tendríamos, conforme al gráfico 1 , dos disposiciones de eventos, a y

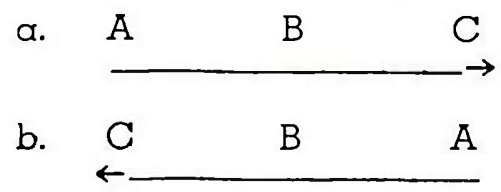

Gráfico 1

(3) En relación con los condicionales contrafácticos véase: Nelson Goodman: "The Problem of Counterfactual Conditionals", incluido en Fact. Fiction and Forecast, Cambridge, Harvard University Press, 1955. También: Ernest Nagel, The Structure of Science, New York, Harcourt, Brace and World, 1961 , pp. 68 ss.

(4) Ver Hans Reichenbach, Op. cit., pp. 70 ss.

(5) Ibíd., pp. 72 ss. 
$b$ en el gráfico, coincidentes en cada caso en un punto cualquiera dado como presente y no tendríamos base suficiente para, desde el punto de vista de uno cualquiera de los procesos, hablar del otro proceso entendiéndolo como poseedor de un sentido temporal negativo, pues para cada evento considerado presente en una de las secuencias se tendría un evento correspondiente en la otra, siendo imposible distinguir lo propuesto en el gráfico 1 de lo mostrado en el gráfico 2, pues en cada caso se darían como presentes AC,

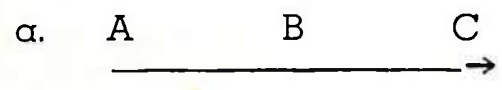

\section{Gráfico 2}

BB, CA (designar a cada secuencia como " $a$ " o "b" es algo puramente accidental).

Conforme al gráfico 2, resulta más apropiado pensar que ambas secuencias representan procesos de una misma temporalidad "positiva". Más aún, incluso si se tratara de un proceso cuya secuencia fuera $A-B-C-B-A$, podría ésta entenderse así: $A-B-C-B-A$, de modo que estaríamos siempre en una temporalidad "positiva", no siendo necesario admitir algo" tan extraño como un regreso al pasado. Es preferible este modo de pensar descartando imaginarios retornos al pasado, pues el suponerlos introduce subrepticiamente la noción de un tiempo en sí (absoluto) en donde los procesos pudieran desenvolverse hacia adelante 0 hacia átrás, y en donde, en consecuencia, pudiera ser posible volver al pasado.

Si eliminamos el supuesto de un tiempo en sí nos encontramos con que no hay un marco de referencia respecto del cual avanzar o retroceder.

De otro lado, resulta importante señalar que puesto que procesos con una temporalidad "negativa" podrían serlo sólo respecto de procesos con temporalidad "positiva" y viceversa, si se pensara que sobre esta base se habla de "tiempo positivo" y "tiempo negativo" y luego se afirmase que no se justifica, por lo anteriormente indicado, hablar de procesos en un "tiempo negativo", se advertiría inmediatamente que hablar de procesos en un "tiempo positivo" resultaría igualmente injustificado, pues no tendrían en re. lación con que serlo.

No parecería, por tanto, correcto pensar, sin más, en procesos en un "tiempo positivo". Es claro que si se hablara sistemáticamente de los procesos como dados en un "tiempo positivo" estaríamos indebidamente introduciendo la noción de un tiempo en sí; ocurriría así por cuanto al no admitir procesos con una temporalidad "negativa", en relación con los cuales otros fuesen "positivos", 
se requeriría al menos un marco de referencia respecto del cual los procesos se desarrollasen "positivamente". La expresión "temporalidad positiva" no debe considerarse por tanto en el sentido arriba mentado, sino como una declaración del reconocimiento del efectivo darse de la temporalidad en el proceso, como un modo de ser específicamente suyo.

De otra parte, lo dicho no significa que se esté indiscriminadamente mezclando y confundiendo las expresiones "tiempo positivo" y "tiempo negativo" al estar empleándose nociones operantes en diferentes niveles, es decir, "tiempo negativo" como lo enteramente inverso respecto de un "tiempo positivo", de un lado, y, de otro lado, "temporalidad positiva" como el efectivo darse de la temporalidad en el proceso. En verdad, "tiempo positivo" y "tiempo negativo" serían (en el caso de hablarse así) ambos descripción del efectivo darse de la temporalidad en el proceso; en este sentido, y sólo en éste, son ambos "positivos"; es a esta "positividad" a la que nos referíamos al sostener que la temporalidad es "positiva". Es obvio que aquí estamos empleando el sentido señalado en segundo lugar y no hay motivo alguno para pensar que se está presuponiendo un tiempo en sí.

Por lo demás, en cuanto se piensá en tiempos "positivo" y "negativo" como un desenvolverse los procesos hacia adelante o hacia atrás, sí se está presuponiendo un tiempo en sí, y es claro el uso del sentido primeramente señalado.

No se supera esta última situación si se pretende considerar estos tiempos "positivo" y "negativo" como resultantes de la simple referencia de un proceso ta otroconsiderado su inverso (hablando de procesos cuyas secuencias están inversamente dispuestas), pues el resultado final de esta posición será el abandono del primer sentido y la adopción del segundo, pues referir un proceso a otro sólo es posible al admitir, explícitamente o no, el desarrollo paralelo de ambos y, naturalmente, el carácter "positivo" de su temporalidad en el segundo sentido (conforme a lo dicho con respecto al gráfico 2).

Sobre la base de lo que antecede consideramos que no es legítimo pretender operar con la noción de un "tiempo negativo", por lo menos no en cuanto se refiere a las afirmaciones hechas acerca de la inmodificabilidad del pasado.

Podemos volver ahora a la cuestión pendiente respecto de la intervención con $X$ en el proceso cuya secuencia quedaba representada en $A-B-C-D$, sin la intervención, y $A-B-C X-$ $E$, con la intervención. La idea es simple: si no se hubiera producido la intervención se hubiera producido l, pero como se llevó a cabo la intervención ocurrió 2 . Cualquiera puede sentirse aquí tentado a reconocer, al parecer sin mayores dificultades, que ha sido probado el contrafáctico (según lo dicho más arriba) y 
consecuentemente ha quedado establecido el hecho de la intervención en el proceso. Sin embargo, es indudable, por otro lado, que se está procediendo con la idea de que se habla del mismo proceso, pues sólo sobre esta base podría pensarse que $\mathrm{X}$ es verdaderamente intervención en un proceso. En este sentido, l y 2 serían el mismo proceso, quedando la única diferencia entre ambos establecida por la intervención de $\mathrm{X}$ en 2 . Es el caso que ocurre $1, y$ no ocurre 2, si no hay intervención; se produce 2 , y no 1 , si hay intervención. Es únicamente en estos términos que puede interpretarse 1 como el proceso sin intervención y 2 como el resultado de haber intervenido con $\mathrm{X}$ en $\mathrm{l}_{\text {; }}$ asimismo, $\mathrm{Y}$ por las mismas razones, puede pensarse una vez ocurrido 2 , lo que hubiese sucedido si no se intervenía con $\mathrm{X}$.

Consideramos, no obstante, que en todo esto existe un error. En verdad no se interviene en un proceso, en todo caso se es ya parte de él. En la secuencia $A-B-C-D$ cada evento es un componente del proceso; igualmente, $A-B-C X-E$ cada evento es un componente del correspondiente proceso. Ahora bien, si $A-B-C-D$ y $A-B-C X-E$ son secuencias que, como tales, $y$ con todos sus elementos, representan procesos, a su vez, con todos sus correspondientes eventos, entonces se deberá reconocer que $A-B-C-D$ y $A-B-C X-E$ representan dos diferentes procesos. En estas circunstancias resulta evidente que lo que llamamos intervención, es decir X en 2 , es en verdad ur. elemento imprescindible en la secuencia $A-B-C X-E$, sin el cual ésta no sería como tal verdaderamente posible. Como cada proceso (en los dos esquemas propuestos) es diferente del otro, no es apropiado decir que uno es el resultado de la intervención en el otro, pues si tal proceso está ya desarrollado y concluido, no puede decirse en modo alguno que se está actuando sobre él; y si tal proceso no está desarrollado ni concluido, será claramente absurdo pensar que se está interviniendo en él; por último, si el proceso no está concluido, pero se está desarrollando, tampoco tendrá mucho sentido decir que se está interviniendo en él, si eso que llamamos intervención es ya necesariamente parte constitutiva, e imprescindible, de él, pues en caso contrario no sería tal; por lo demás, lo relativo a la situación de sus eventos últimos, los no alcanzados aún en el desarrollo del proceso, queda incluido en lo dicho en el caso anterior. En realidad, sólo en el terreno de la imaginación, en donde demasiadas cosas son posibles, cabe pensar lo que sería un proceso sin la "intervención" en éli pero esto es equivalente a pensar lo que sería un proceso en el que uno cualquiera de sus eventos no ocurriera ( $\sin$ que por esto se tenga que pensar en términos de no intervenciones). Lo más que cabe aquí es hacer conjeturas basadas en comparaciones con otros procesos, imaginando, en relación con ellos, la forma que en determi- 
nadas condiciones tomaría un proceso; pero las conjeturas no son los hechos (en tanto se las tome como tales).

La confusión es clara. El proceso 2 no es una consecuencia de una intervención en el proceso l, se trata en realidad de un proceso diferente en el cual uno cualquiera de sus eventos constitutivos es considerado intervención; pero si se trata de un proceso distinto, entonces no resulta pertinente decir que se ha intervenido en otro proceso para desarrollar el proceso en mención; lo más que puede decirse es que se es parte de un proceso particular, teniendo éste, ocasionalmente, algunas semejanzas con otros procesos.

De acuerdo con lo visto, la afirmación hecha en el sentido de que es posible cambiar el futuro mediante la intervención en los procesos es, en los términos en que corrientemente se formula, insostenible; pues ¿cómo puede ser cambiado el futuro? Esto es algo incorrecto si tiene que ser literalmente entendido. Sólo presuponiendo o admitiendo de algún modo su existencia, pensando quэ el futuro será tal o cual, puede concebirse la intervención como lo que hace que éste sea otro (6). Pero si el resultado iba a ser ese otro ¿qué era lo que iba a ocurrir como tal y cual y que pensábamos en cambiar?

Es patente que hay en todo esto un error, y lo extraño de la situación descrita no es sino el resultado de él. El error no se produce si pensamos en los términos antes señalados.

Es obvio que en el proceso 2 el evento final (en los términos

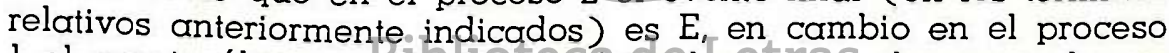
1 el evento último es D. Siendo éste el caso, en el proceso l partiendo de $A$ el futura será Dumientráscque en el proceso 2, siempre partiendo de $A$, el futuro será $E_{i}$ no resulta en absoluto claro en qué sentido puede decirse (con un sentido no meramente metafórico, se entiende) que se ha modificado, o que se va a modificar, el futuro. Es evidente que tratóndose de procesos diferentes se alcanzará en cada caso un futuro diferente, pero nadie diría que a partir de 1 se llegó al futuro de 2, por ejemplo; esto último sería cosa de imposibilidad.

Ahora, si partiendo de A se concluye en $D$, entonces se alcanzó el futuro correspondiente al proceso l, y si se llegó a $E$, se alcanzó el futuro de 2; y no es el caso de que se pueda pensar que el futuro de $l$ era $D$, pero se llegó a $E$, pues esto sería manifiestamente erróneo, dado que si $\mathrm{D}$ era el futuro correspondiente a $\mathrm{l}$, entonces lo era y no podía no serlo; si D tenía que ocurrir, tenía que

(6) Algunas consideraciones similares a las aquí tratadas pueden encontrarse en A. J. Ayer, El problema del conocimiento, Buenos Aires, Editorial Universitaria de Buenos Aires, 1962, pp. 197 ss. 
ocurrir; lo mismo vale respecto de E. No admitir esto trae como consecuencia caer en la insólita situación de tener que sostener que algo será, pero no será; posición insostenible, indudablemente, por contradictoria.

Pensar que el futuro puede ser cambiado equivaldría, de acuerdo con lo dicho, a decir que, respecto del proceso 1, por ejemplo, D ocurrirá, pero no ocurrirá, y ambas cosas con carácter de efectividad, por supuesto.

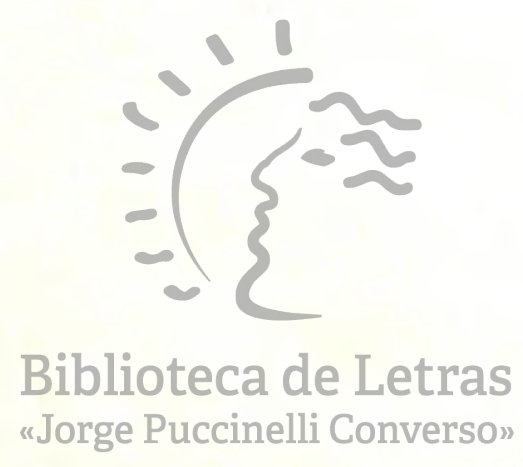

